\title{
Mirror symmetry of higher order aberrations between right and left eyes
}

\author{
M Oberholzer*, WDH Gillan ${ }^{\dagger}$ and A Rubin ${ }^{\dagger}$
}

*Department of Optometry, School of Allied Health Professions, Faculty of Health Sciences, University of the Free State, PO Box 339, Bloemfontein, 9300 South Africa

†Department of Optometry, Faculty of Health Sciences, University of Johannesburg, PO Box 524, Johannesburg, 2006 South Africa

†< oberholzerm@ufs.ac.za >

Received 29 November 2013; revised paper accepted 17 March 2014

\begin{abstract}
Introduction: There is evidence that certain measures of visual function show some type of relationship between right and left eyes in the same individual. Similarly, particular ocular maladies may be related, or be symmetric, in the right and left eyes of the same person. There is also evidence to suggest that certain relationships do not exist between eyes in an individual. For example, diseases such as glaucoma are often asymmetric in their progression in the two eyes of an afflicted individual. Inter-ocular mirror symmetry between right and left eyes, when considering ocular wavefront aberrations (WAs), has been shown to exist by some authors. This study investigates whether mirror symmetry of high order aberrations (HOAs) exists between the right and left eyes in a cohort of South African subjects.

Method: Third to $5^{\text {th }}$ order Zernike coefficients
\end{abstract}

(HOAs) were measured on both eyes of 66 subjects (132 eyes) using a Zywave aberrometer. A total of 15 Zernike coefficients for each eye were obtained. Mirror symmetry was investigated using correlation coefficients between the various measurements obtained from each eye.

Results: Pearson product-moment correlation coefficients provide evidence that the majority of the 15 Zernike coefficients suggest the presence of mirror symmetry between right and left eyes of the 66 subjects. Examples of individual scatter plots comparing right and left eyes are presented.

Conclusion: The results of this study suggest that mirror symmetry exists in the HOAs obtained from the 132 eyes measured and thus care should be exercised when combining eyes of individuals for analysis. (S Afr Optom 2013 73(1) 39-44)

Key Words: Mirror symmetry, aberrations, interocular mirror symmetry

\section{Introduction}

When analysing measurements obtained from both eyes of an individual one needs to take the correlation that often exists between the two eyes into account, because the right and left eyes of individuals are more likely to be similar to each other than measurements obtained from an unrelated person ${ }^{1}$. Also, some conditions are more likely to be present in both eyes while other conditions are more prevalent in one eye

*This paper is based on postgraduate studies of the first author with the supervision of Professors WDH Gillan and A Rubin of the University of Johannesburg 
only. For example: choroidal melanoma only occurs in one eye in $99 \%$ of cases while blepharitis occurs in both eyes in $95 \%$ of cases ${ }^{2}$. It is also known that some degree of symmetry exists between the right and left eyes of individuals when considering refractive state ${ }^{3}$. Smolek et $a l^{4}$ have expressed concern that not accounting for the existence of mirror symmetry between right and left eyes can invalidate results of studies investigating aberrations. Brown et $a l^{5}$ state that mirror symmetry between right and left eyes of individuals is a problem that needs to be addressed when the HOA data of right and left eyes of individuals are compared or when the HOA data of right and left eyes of individuals are combined. To allow for the possible existence of mirror symmetry between right and left eyes one may either use only right or only left eyes for a study, or right and left eyes are analysed separately or one may adjust the signs of some of the Zernike coefficients for one of the eyes investigated, usually the left eye ${ }^{2}$.

Numerous authors ${ }^{6-12}$ have shown evidence supporting the existence of mirror symmetry between the right and left eyes of the same individual. In contrast to the findings of these studies, Howland and Howland ${ }^{13}$ found that WAs differ between subjects and are seldom symmetrical. Mirror symmetry between right and left eyes of individuals can be assessed by various methods. Marcos and Burns ${ }^{10}$ calculated correlation coefficients of individual Zernike coefficients between right and left eyes after reversing the sign of odd symmetric terms in the right eye. The authors ${ }^{10}$ found that five of the 12 subjects used in their study showed a statistically significant prevalence of mirror symmetry between the right and left eyes. Thibos et $a l^{7}$ investigated correlation coefficients for Zernike coefficients that have even symmetry about the vertical axis (should have a positive correlation) separately from those that have odd symmetry about the vertical axis (should have a negative correlation). The authors $^{7}$ showed support for the presence of mirror symmetry in their subjects. Castejón-Mochón et al ${ }^{12}$ investigated the correlations for defocus, astigmatism and 4th order spherical aberration in 35 pairs of eyes. The results of their study suggested a slight tendency towards mirror symmetry. Porter et al ${ }^{8}$ measured the WAs of right and left eyes under natural accommodation for $5.7 \mathrm{~mm}$ pupils in a sample of 109 subjects and correlation coefficients were calculated for individual
Zernike coefficients. Porter et $a l^{8}$ argue: "to have perfect mirror symmetry between right and left eyes, radially symmetric Zernike modes and those dependent on an odd power of $y$ need to be positively correlated in both eyes, while polynomials that are dependent on an odd power of $x$ should be negatively correlated in both eyes". Thus, to have perfect mirror symmetry, negative correlation coefficients would be expected for Zernike polynomials $Z_{2}^{-2}, Z_{3}{ }^{1}, Z_{3}^{3}, Z_{4}^{-2}, Z_{4}^{-4}, Z_{5}{ }^{1}, Z_{5}^{3}$ and $Z_{5}^{5}$. The correlations were calculated for each Zernike coefficient individually for the whole sample of 109 eyes with no sign adjustments made to either eye. The results showed that $75 \%$ of the investigated Zernike coefficients $\left(1^{\text {st }}\right.$ to $5^{\text {th }}$ orders $)$ were significantly correlated across the eyes. These significant correlation coefficients between the Zernike coefficients confirm a form of symmetry between the right and left eyes. A positive correlation coefficient occurs when a Zernike coefficient in one eye (of a sample) increases as the coefficient in the other eye also increases. A negative correlation coefficient again occurs when the Zernike values in one eye increase as the coefficient in the other eye decreases in value. But as mentioned, Howland and Howland ${ }^{13}$, found that WAs differ greatly between subjects and are seldom symmetrical. The aim of this study was to investigate whether mirror symmetry possibly exists between the right and left eyes of 66 individuals.

\section{Methods}

This was a retrospective study and all data were sourced from six different ophthalmic surgeons' case notes. All measurements were obtained in the same environment using the same instruments which were operated by a single technician. The subjects chosen had all been examined for possible Laser-Assisted in situ Keratomileusis (LASIK) eye surgery in 2009 and 2010 and included both males and females of any age. To be included in the study, subjects had to have ceased contact lens wear for at least one week prior to any measurements being taken. Also, myopic astigmats with a spherical component of $(0$; -7] $\mathrm{D}$ (thus excluding $0 \mathrm{D}$ and including $-7 \mathrm{D}$ ) and a cylinder (astigmatic) magnitude greater than zero were included in the study. Subjects were excluded if they had a history of previous ocular surgery, current ocular pathology or a history of corneal disease, 
abnormalities or ectasia. The total sample size in this study was 66 subjects (132 eyes).

Measurements were firstly taken by the technician with an Orbscan II (software version 3.14) followed by further measurements with a Zywave aberrometer (software version 5.2). The range of spherical powers for the right eye group was $[-0.11 ;-6] \mathrm{D}$ and for the left eye group, $[-0.01 ;-7] \mathrm{D}$ whereas the range of the cylinder powers for the right eyes was $[-0.05$; -4.97] $\mathrm{D}$ and for the left eyes $[-0.06 ;-5.12] \mathrm{D}$. All measurements were taken with the eyes in their natural, undilated state. Pupil sizes varied under the selected lighting conditions but were all converted to the same $6 \mathrm{~mm}$ pupil size by the Zywave aberrometer. The measurements presented by the Zywave aberrometer include the $3^{\text {rd }}$ to $5^{\text {th }}$ order Zernike coefficients for $6 \mathrm{~mm}$ pupil diameters. Correlation coefficients were performed using Statistica version 10 statistical software packages. Ethics approval was granted by the Ethics Committee of the University of Johannesburg, South Africa. (No pilot study was conducted as the data was retrospective in nature.)

\section{Results}

The ages of the subjects ranged between 14 and 66 years. Pearson product-moment correlation coefficients between the right and left eyes for each of the 15 individual higher order Zernike coefficients ( $3^{\text {rd }}$ to $5^{\text {th }}$ order) of the 66 subjects were calculated. Table 1 shows the correlation coefficients for each individual Zernike coefficient. Coefficients expected to have negative correlations are indicated with an asterisk ( $p$ - values are included in the table). Whether a correlation coefficient was found to be significant or contribute to the existence of possible symmetry is included in Table 1 (column four indicates whether the correlation coefficient between the right and left eyes of each of the stated coefficients contributes to mirror symmetry according to Porter et $a l^{8}$ ).

Table 1: Correlation coefficients for each Zernike coefficient for a $6 \mathrm{~mm}$ pupil, between right and left eyes are shown. In column 3 , bold type indicates significant correlations. Asterisks indicate the Zernike coefficients expected to have negative correlations.

\begin{tabular}{|l|l|l|l|}
\hline $\begin{array}{l}\text { Zernike coef- } \\
\text { ficient }\left(3^{\text {rd }} \text { to } 5^{\text {th }}\right. \\
\text { order })\end{array}$ & Correlation coefficient & $\begin{array}{l}\text { Significant (S) or In- } \\
\text { significant (IS) }\end{array}$ & $\begin{array}{l}\text { Contributes to mirror } \\
\text { symmetry }\end{array}$ \\
\hline$C_{3}{ }^{-3}$ & $0.68(p=0.00)$ & $\mathbf{S}$ & Yes \\
\hline$C_{3}{ }^{-1}$ & $0.92(p=0.00)$ & $\mathbf{S}$ & Yes \\
\hline$C_{3}{ }^{1} *$ & $-0.82(p=0.00)$ & $\mathbf{S}$ & Yes \\
\hline$C_{3}{ }^{3} *$ & $-0.67(p=0.00)$ & $\mathbf{S}$ & Yes \\
\hline$C_{4}{ }^{-4} *$ & $-0.46(p=0.00)$ & $\mathbf{S}$ & Yes \\
\hline$C_{4}{ }^{-2} *$ & $-0.05(p=0.67)$ & $\mathrm{IS}$ & Yes \\
\hline$C_{4}{ }^{0}$ & $0.84(p=0.00)$ & $\mathbf{S}$ & Yes \\
\hline$C_{4}{ }^{4}$ & $0.66(p=0.00)$ & $\mathbf{S}$ & Yes \\
\hline$C_{4}{ }^{-5}$ & $\mathbf{S}$ & Yes \\
\hline$C_{5}{ }^{-3}$ & $0.49(p=0.00)$ & $\mathrm{IS}$ & Yes \\
\hline$C_{5}{ }^{-1}$ & $0.003(p=0.98)$ & $\mathbf{S}$ & Yes \\
\hline$C_{5} C_{5}{ }^{1} *$ & $0.36(p=0.003)$ & $\mathbf{S}$ & Yes \\
\hline$C_{5}{ }^{3} *$ & $0.65(p=0.00)$ & IS & Yes \\
\hline$C_{5}{ }^{5} *$ & $-0.10(p=0.45)$ & IS & Yes \\
\hline
\end{tabular}




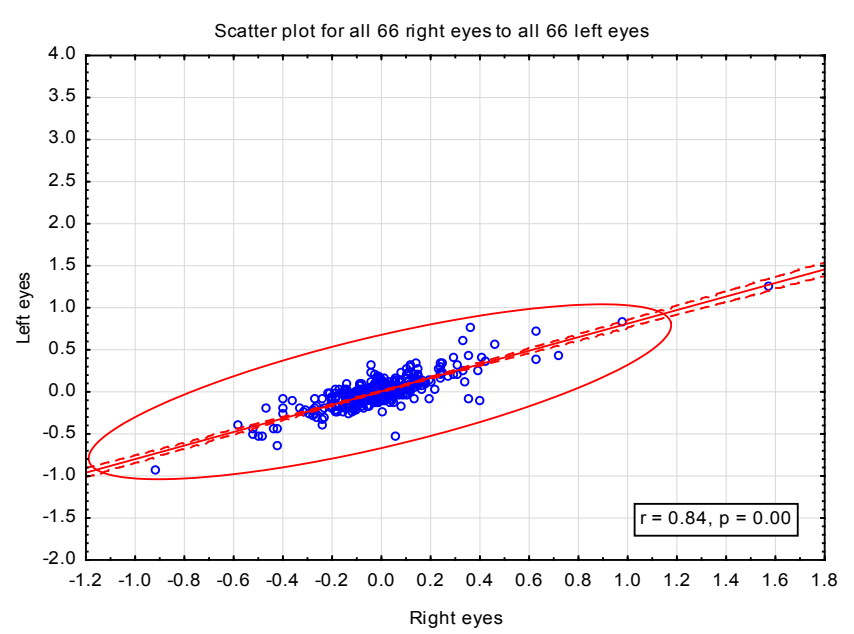

Figure 1: A scatter plot presenting the 66 right eyes against the 66 left eyes for the following coefficients: $C_{3}{ }^{-3}, C_{3}{ }^{-1}, C_{4}{ }^{0}, C_{4}{ }^{2}$, $C_{4}^{4}, C_{5}{ }^{-5}, C_{5}{ }^{-3}$ and $C_{5}{ }^{-1}$. Units are in microns. According to Porter et $\mathrm{al}^{8}$ positive correlations between right and left eyes in these coefficients should be expected if mirror symmetry exists between the two eyes. A possible outlier is seen outside the $95 \%$ confidence ellipse.

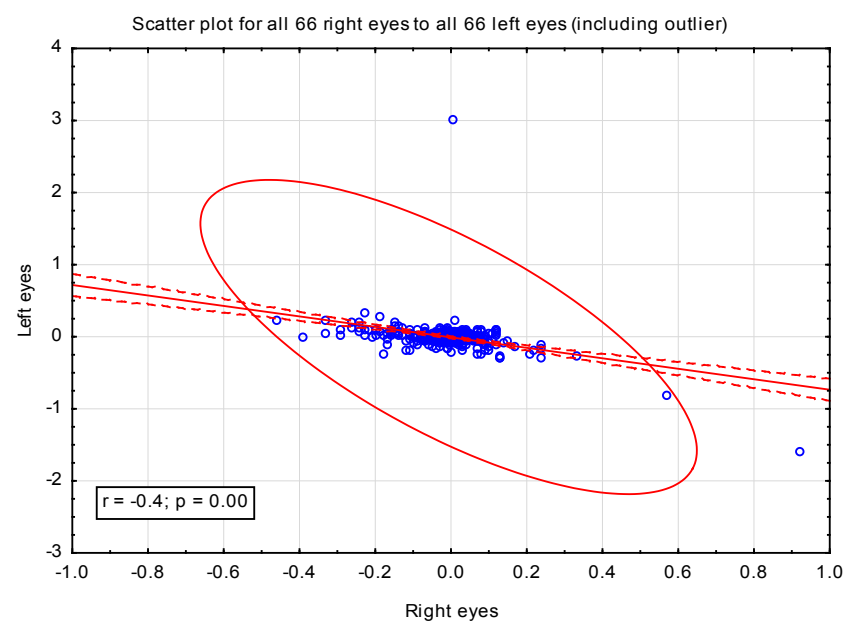

Figure 2: This scatter plot represents the right eyes against the left eyes for the following coefficients: $C_{3}{ }^{1}, C_{3}{ }^{3}, C_{4}{ }^{-4}, C_{4}{ }^{-2}, C_{5}{ }^{1}$, $C_{5}{ }^{3}$ and $C_{5}{ }_{5}^{5}$. Porter et al $^{8}$ argue that negative correlations between right and left eyes for these coefficients should be expected if mirror symmetry exists between the two eyes. Two possible outliers are observed outside the $95 \%$ confidence ellipse.

The scatter plot in Figure 1 combines all the coefficients (of 66 subjects) that would be expected to have positive correlations if mirror symmetry was present between right and left eyes. The correlation coefficient is $0.84(p=0.00)$ and represents a strong positive correlation between the coefficients for the right and left eyes. In Figure 2, a similar plot for all the coefficients which would be expected to have a negative correlation if mirror symmetry was present is shown. The correlation coefficient for the data shown in Figure 2 is $-0.4(p=0.00)$, a weak, negative, yet significant, correlation. In both figures (1 and 2) $95 \%$ confidence intervals on the regression line, as well as 95\% confidence ellipses for the data, are shown.

In the interests of brevity, Figures 3 and 4 show examples of 66 single coefficients $\left(C_{3}{ }^{-1}\right.$ in Figure 3 and $C_{3}{ }^{3}$ in Figure 4) which show positive and negative correlations respectively. According to Porter et $a l^{8}$, specific Zernike coefficients should be positively (or negatively) correlated in the presence of mirror symmetry and Figures 3 and 4 show two such correlations. The correlation coefficients and $p$ values are indicated in the relevant figures.

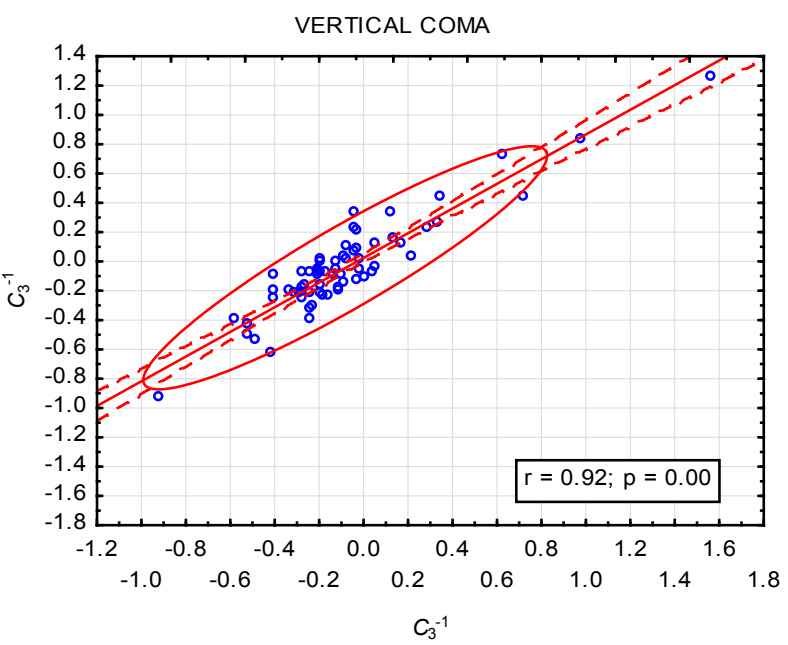

Figure 3: The scatter plot for the right and left eyes of 66 subjects for coefficient $C_{3}{ }^{-1}$ with a positive regression line and significant correlation coefficient of 0.92 . Units are microns. The 95\% confidence interval (dashed lines) is also included with a $95 \%$ confidence ellipse. Several measurements are seen outside the $95 \%$ confidence ellipse.

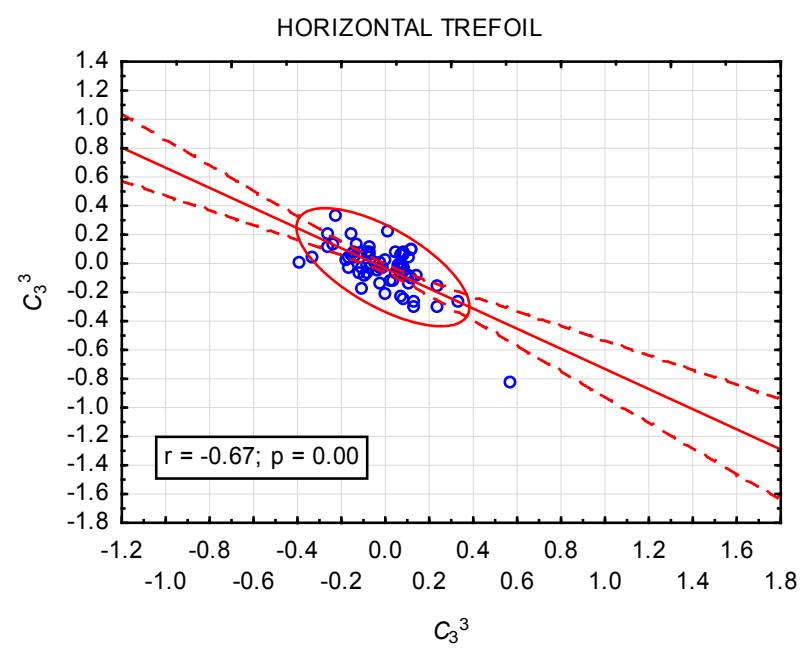

Figure 4: A scatter plot for the $C_{3}{ }^{3}$ coefficients of the right and left eyes of 66 subjects. A negative regression line and narrow confidence region are presented for $C_{3}{ }^{3}$ in this scatter plot. Two measurements are seen outside the $95 \%$ confidence ellipse. 


\section{Discussion}

Table 1 shows the correlation coefficients and related data for the 15 Zernike higher order coefficients evaluated in this study. Porter et $a l^{8}$ have stated that it would be expected for Zernike coefficients $C_{3}{ }^{1}, C_{3}{ }^{3}$, $C_{4}^{-2}, C_{4}^{-4}, C_{5}{ }^{1}, C_{5}{ }^{3}$ and $C_{5}{ }^{5}$ to have negative correlations between right and left eyes, while the remaining eight coefficients (in this case $C_{3}^{-3}, C_{3}^{-1}, C_{4}^{0}, C_{4}^{2}, C_{4}^{4}$, $C_{5}^{-5}, C_{5}^{-3}$ and $C_{5}^{-1}$ ) should have positive correlations between right and left eyes if mirror symmetry was present. All correlation coefficients (except for $C_{5}{ }^{5}$ ) show the relevant type of correlation (positive or negative) to support the presence of mirror symmetry which concurs with the findings of mirror symmetry in the study sample of Porter et $a l^{8}$. However, not all the correlation coefficients have been shown to be significant (see Table 1). Figure 1 presents a scatter plot of the eight coefficients that should show positive correlations for the sample of 66 subjects combined. All corresponding coefficients for the right and left eyes were plotted against each other and a significant positive correlation coefficient of 0.84 was found ( $p=0.00)$, suggesting that mirror symmetry is present. Similarly, Figure 2 shows the remaining seven Zernike coefficients that should have negative correlations (Porter et $a l^{8}$ ) for perfect symmetry and the correlation coefficient $(r=-0.40)$ is significant $(p=0.00)$. When assessing whether mirror symmetry is present between right and left eyes, Thibos et $a l^{7}$ similarly pooled all Zernike coefficient data that should be positively (or negatively) correlated, determined the correlation coefficient between right and left eyes and presented the data in a scatter plot. The results of this study support the findings of Thibos et $a l^{7}$.

Scatter plots for each of the 15 Zernike coefficients were plotted to evaluate the possible presence of mirror symmetry within the data of the 66 subjects (only two examples of the 15 plots, namely Figures 3 and 4 are included here). If mirror symmetry is present, then negative correlation coefficients would be expected for the following Zernike coefficients: $C_{3}{ }^{1}, C_{3}{ }^{3}, C_{4}{ }^{-2}, C_{4}{ }^{-4}$, $C_{5}{ }^{1}, C_{5}{ }^{3}$ and $C_{5}{ }^{5}$. In this study, six of these coefficients showed negative correlations (see Table 1). However, of these, not all the correlations are significant. Figure 4 shows an example of negative correlation, in this case for $C_{3}^{3}$. The eight coefficients (Porter et $a l^{8}$ ) that should present with positive correlations between the two eyes are: $C_{3}^{-3}, C_{3}^{-1}, C_{4}^{0}, C_{4}^{2}, C_{4}^{4}, C_{5}^{-5}, C_{5}^{-3}$ and $C_{5}^{-1}$ and Figure 3 shows an example for $C_{3}^{-1}$. The results of this study show that all eight Zernike coefficients (that should show positive correlations) support the presence of mirror symmetry. These results also support numerous other reports that have suggested that mirror symmetry exists between right and left eyes $^{6-8,10,12}$ in the same individual. Thus one needs to be careful in combining HOA data for the right and left eyes of the same individual. Analysis should be done with the eyes considered separately or one might randomly select either the right or the left eye only of study participants for inclusion in a single sample for further analysis but where nessecary the signs of some coefficients would need to be changed.

\section{Conclusion}

Mirror symmetry is suggested between the right and left eyes of the same individual for this sample of 66 subjects ( $n=132$ eyes). The calculated correlation coefficients between the right and left eyes indicate that most of the 15 Zernike coefficients in the right and left eyes contribute to mirror symmetry. Eyes should thus be used separately in sample groups when investigating HOAs or alternatively, some Zernike coefficients of either eye should be altered to compensate for mirror symmetry before combining right and left eye data together into one sample for analysis. A possible limitation here is that the study only included a clinical population who were mostly interested in having LASIK. However, a strength of this study is that it brings another dimension to research in the field of ocular wavefront aberrations as it includes both the spherical as well as the astigmatic components of refractive error and is presented in a South African context. The exploration of mirror symmetry between the right and left eyes of the same subject in the astigmatic myopic groups defined within this study is, to the best of our knowledge, unique and contributes toward a better understanding of the mirror symmetry of HOAs in especially astigmatic myopes. A possible future research suggestion includes a closer look into the Zernike coefficients that contribute more profoundly to mirror symmetry between right and left eyes. 


\section{References}

1. Karakosta A, Vassilaki M, Plainis S, Elfadl NH, Tsilimbaris M, Moschandreas J. Choice of analytic approach for eye-specific outcomes: one eye or two. Am J Ophthalmol 2012153 571-579.

2. Murdoch IE, Morris SS, Cousens SN. People and eyes: statistical approaches in ophthalmology. $\mathrm{Br} \mathrm{J}$ Ophthalmol 199882 971-973.

3. McKendrick AM, Brennan NA. The axis of astigmatism in right and left eye pairs. Optom Vis Sci 199774 668675.

4. Smolek MK, Klyce SD, Sarver EJ. Inattention to nonsuperimposable midline symmetry causes wavefront analysis error. Arch Ophthalmol 2002120 439-447.

5. Brown WL, White T, Capelli G, Rosenberg R. American National Standards Institute: American National Standard for Ophthalmics - Methods for Reporting Optical Aberrations of Eyes. 2010: Z80.28-2010.

6. Liang J, Williams DR. Aberrations and retinal image quality of the normal human eye. J Opt Soc Am A 1997 14 2873-2883.

7. Thibos LN, Hong X, Bradley A, Cheng X. Statistical variation of aberration structure and image quality in a normal population of healthy eyes. J Opt Soc Am 2002 19 2329-2348.

8. Porter J, Guirao A, Cox IG, Williams DR. Monochromatic aberrations of the human eye in a large population. J Opt Soc Am A 200118 1793-1803.

9. Bao J, Le R, Wu J, Shen Y, Lu F, He JC. Higher-order wavefront aberrations for populations of young emmetropes and myopes. J Optom 20092 51-8.

10. Marcos S, Burns SA. On the symmetry between eyes of wavefront aberration and cone directionality. Vis Res $2000402437-2447$.

11. Wang L, Koch DD. Ocular higher-order aberrations in individuals screened for refractive surgery. $J$ Cat Refract Surg 200329 1896-1903.

12. Castejón-Mochón JF, López-Gil N, Benito A, Artal P. Ocular wave-front aberration statistics in a normal young population. Vis Res 200242 1611-1617.

13. Howland HC, Howland B. A subjective method for the measurement of monochromatic aberrations of the eye.

J Opt Soc Am A 197767 1508-1518. 